\title{
Los mapas geopolíticos de la Unesco: entre la distinción y la diferencia están las asimetrías. El éxito (exótico) del patrimonio inmaterial ${ }^{1}$
}

\section{UNESCO's geopolitical maps: The lack of symmetry lies between distinction and difference. The (exotic) success of Immaterial Heritage}

\author{
Beatriz SANTAMARINA CAMPOS \\ Departamento de Sociología y Antropología Social \\ Universidad de Valencia \\ Beatriz.Santamarina@uv.es
}

Recibido: 2 de mayo de 2012

Aceptado: 14 de abril de 2013

\section{Resumen}

El patrimonio inmaterial es una categoría política confusa y contradictoria. La democratización y universalización del patrimonio cultural ha permitido su profusión y revalorización. Ahora bien, pese a su actual protagonismo, su construcción responde tanto a las viejas demarcaciones de antaño como a un ejercicio globalizado de simetrías aparentes. Es decir, del colonialismo al nuevo imperialismo parece que las cosas no han cambiado sustancialmente. Así, podemos afirmar que hemos asistido a un 're-bautizo' de lo antes llamado científicamente 'folck-lore'. Y en ese cambio de la concepción patrimonial hemos pasado: de poner el acento en la distinción a poner el énfasis en la diferencia; de la perseguida conservación a la necesidad de la salvaguardia; del acento en el resultado al énfasis en el proceso; del confinamiento político-científico de la definición a la voluntad de subrayar la participación y la comunidad. En este artículo pretendemos acercamos al nicho normalizado del patrimonio inmaterial, atendiendo a su institucionalización, discurso y praxis hegemónica. Para ello realizaremos un análisis conceptual como marco para atender a su edificación y su plasmación en un particular mapa geopolítico.

Palabras clave: Patrimonio inmaterial, salvaguardia, diferencia, comunidad, dominación, poder.

${ }^{1}$ Este trabajo se enmarca en el proyecto Patrimonializacion y Redefinicion de la Ruralidad. Nuevos Usos del Patrimonio Local (CSO2011-29413) financiado por el Ministerio de Economia y Competitividad. Una primera versión se presentó en el XII Congreso Nacional de Antropología, esta ha sido considerablemente ampliada y revisada. Agradezco a Eliseu Carbonell y, sobre todo, a Camila del Mármol sus generosos comentarios. 


\begin{abstract}
Immaterial Heritage is a political category that is both confusing and contradictory. The democratization and universalization of Cultural Heritage have made the latter more profuse and have led to its increased importance. Even so, and despite its current levels of protagonism, its construction responds not only to outmoded parameters but also to a global exercise in apparent symmetry. In other words, things do not appear to have changed significantly from Colonialism to the new Imperialism. So it is safe to say that we are now privy to a "re-christening" of what was formerly referred to as "folklore". And in this change in the conception of heritage we have gone from putting the accent on distinction to placing it on difference; from the pursuit of conservation to the need for safeguarding; from underscoring results to emphasizing process; from confining ourselves to a political-scientific definition to the will to underline participation and community. This article sets out to approach the normalized niche of Immaterial Heritage, looking at its institutionalization, its discourse and its uniform practice. To do so, we will carry out a conceptual analysis as a framework aimed at constructing it and placing it on a particular geopolitical map.
\end{abstract}

Key words: Intangible heritage, safeguarding, difference, community, domination, power

Referencia normalizada: Santamarina Campos, B. (2013) Los mapas geopolíticos de la Unesco: entre la distinción y la diferencia están las asimetrías. El éxito (exótico) del patrimonio inmaterial, en Revista de Antropología Social, 22: 263-286.

SUMARIO: 1. Introducción. 2. Desnudando la ortodoxia. 3. Normativizando lo intangible como natural. 4. Del occidentalismo al orientalismo. 5.Conclusiones. 6. Referencias bibliográficas.

\title{
1. Introducción
}

Hablar del patrimonio inmaterial o intangible ${ }^{2}$, e incluso de patrimonio oral, es, sin duda, hablar de una demarcación en sí misma confusa y contradictoria y, como no podría ser de otro modo, esencialmente política. Y podríamos añadir más, sólo desde un análisis de la política patrimonial y de lo político en el patrimonio es posible llegar a comprender algo de ese ejercicio metacultural de translocalización — patrimonio de la humanidad - y recontextualización (Kirshenblatt-Gimblett, 2004) de las geopolíticas de los procesos patrimoniales — dejaremos al margen la inconsistencia de un pretendido patrimonio, en sí mismo, universal- . Producción metacultural reificada, por ser más finos, que genera diferencias y desigualdades a partir de una jerarquización global de valores (Herzfeld, 2004) o de un sistema global taxonómico e institucionalizado (Palumbo, 2010). Durante las últimas décadas, gracias a la expansión sufrida por el constructo decimonónico de 'patrimonio'3 (Ariño, 2002; Santamarina, 2005), hemos asistido al reconocimiento global y globalizado

${ }^{2}$ En castellano se utilizan ambas acepciones mientras que los francófonos utilizan la formula de patrimoine culturel immatérielle y los anglófonos la de intangible cultural heritage.

${ }^{3}$ Expansión, según Heinich (2010), que es cronológica, topográfica, categorial y conceptual. Por otro lado, resta señalar aquí que el patrimonio no es una realidad inmanente que se descubre, sino un constructo político marcado por la invención, el pasado y la identidad (Hobsbawm 
de otros patrimonios potenciales que hasta este momento eran o bien no reconocidos como tales o bien minusvalorados - patrimonios menores - . La llamada democratización del patrimonio - la puesta en valor de nuevos referentes y agentes - no deja de ser un proceso confuso de reconocimientos ya conocidos — prácticas- y bien articulados - atribuciones y distribuciones-. Las viejas distinciones, en el sentido bourdieano $(1991,1998)$, antes vestidas de gala, se tornan ahora discretas en un ejercicio globalizado de simetrías aparentes. Es decir, las viejas jerarquizaciones de antaño amparadas en el conocimiento y sacralizadas y consagradas, como ordenes generadores de autenticidad — verdad/poder - , ceden protagonismo aparente para alcanzar el falso mito de la equidad. Del colonialismo al nuevo imperialismo - globalización - parece que las cosas no han cambiado en demasía o, si se prefiere, sustancialmente.

Así, en el caso del patrimonio inmaterial, podemos decir que asistimos a un 're-bautizo' — vieja política discursiva de las prácticas hegemónicas cuando sufren alguna perturbación o crisis- de lo antes llamado científicamente 'folck-lore' - pueblo-conocimiento, saber del pueblo-. Este re-conocimiento no ha hecho más que consolidar viejos modelos colonialistas en un momento de una fuerte demanda patrimonial — avidez de consumo patrimonialista - que produce una inflación patrimonial, en expresión de Heinich (2009), contraria a su propia edificación como bien escaso (Lacarrieu, 2008). Ahora bien, dicha demanda debe ser contextualizada. Por un lado, la contracción espacio-temporal, la aceleración de los acontecimientos y la multiplicación de flujos, comunicaciones y redes, etcétera, han llevado a una intensa interacción cultural provocando procesos tanto de homogeneización, como de desterritorialización, reterritorialización e hibridación (Appadurai, 1996; García Canclini, 1999; Hernández et al., 2005). Frente a los mismos se haría necesario subrayar la heterogeneidad y las diferencias ${ }^{4}$. De ahí que, en la última década, se haya pasado de poner el acento en la distinción - lógica de activación clásica decimonónica - a poner el énfasis en la diferencia - lógica de reconocimiento vigente-. Dicho de otro modo, si la alta cultura tenía entidad sobrada como motor de selección de lo auténtico, hoy la llamada globalización obliga a salvaguardar las diferencias — 'culturales' - en aras del mercado.

Por otro, y unido a lo anterior, hemos asistido a una reestructuración económica, con una terciarización de la economía y una explosión del turismo, para algunos democratización del turismo, para otros imperialismo turístico (Nash, 1992; Esteve, 1983; West y Carrier, 2004). El consumo de lo 'auténtico', en el sentido de lo 'verdadero', mueve hoy cifras millonarias en un turismo cada vez más exigente que busca lo natural — tanto natural como cultural — frente a lo artificial — tanto

y Ranger, 1988; Prats, 1997; Lowenthal, 1998; Iniesta, 1999; Quintero, 2005; Santamarina, 2005).

${ }^{4}$ Para la UNESCO, tal y como reconoce en el preámbulo de la Convención del 2003, la globalización obligaría a tomar medidas para la salvaguardia por el peligro que supone. En este mismo sentido, Arizpe (2004) habla del peligro de la trivialización. Estas posturas dejan de lado los procesos activos de creación y recreación permanente, que permiten redefiniciones constantes y contextuales. 
natural como cultural - como si de depósitos estancos y almacenables se tratara - mercancías - La alianza de turismo y patrimonio se debe, y debe mucho, a los mapas glocalizados de las mercancías de lo auténtico y lo verdadero. Lo que podríamos denominar como marketing heritage que conduce a la introducción de la 'marca' como esencia cultural - léase autenticidad-. Y en esa búsqueda de lo genuino los patrimonios denominados natural e inmaterial han ido ganando la partida al exclusivo y fotogénico patrimonio cultural — patrimonio artístico-histórico - 5 . Ambos han compartido una similar construcción discursiva, basada en criterios científicos (método-lógicos), que los emparentaba y cosificaba —objetivación vía intervención - . De tal forma que, situados en el mismo plano — en o cerca de la naturaleza-, era fácil justificar la relación subordinada y asimétrica frente a la cultura en mayúsculas y singular (Santamarina, 2009). Pensemos en que el folklore fue concebido como el producto natural de la cultura de un grupo (Bortolotto, 2011a).

En el caso que nos ocupa, la naturaleza implícita del patrimonio inmaterial se sitúa, preferentemente, en espacios culturalmente naturalizados — patrimonio rural versus cultura popular tradicional — contagiándose de las virtudes de los mismos a través de restituciones metonímicas ${ }^{6}$ y relaciones metafóricas que consagran nuestros esquemas de categorización. Siguiendo a Frigolé (2007, 2010), se produce un maridaje casi perfecto entre lo rústico, lo salvaje y lo silvestre. En nuestro imaginario colectivo, esta producción responde a la recreación de un pasado cada vez más exigente y distante. Exigente porque se imponen modelos únicos de consumo patrimonial neoliberal, mercantilizando a sujetos y objetos, que responden a tecnologías gubernamentales. De tal forma que la distinción entre parques temáticos y museos, espacios naturales o recreaciones recreacionales — valga la redundancia- es cada vez más costosa. Por dar un ejemplo, siguiendo a Martí (2010), pensemos en el funcionamiento como parque del complejo de la Ciudad de las Artes y las Ciencias de Valencia o por dar otro veamos como presenta la agencia turística valenciana el complejo del Bioparc: 'Valencia al natural, Bioparc, Naturaleza en abierto'. Y distante porque cada vez el pasado está, a la vez, más lejano y más cercano. Echar la vista atrás se ha convertido en un ejercicio de vértigo, hasta tal punto que el pasado puede llegar a ser nuestra sombra pisando nuestro presente. Este último se nos muestra cada vez más fugaz (Kirshenblatt-Gimblett, 2004). De ahí la imperiosa necesidad de patrimonializar (Heinich, 2009).

${ }^{5}$ A lo largo del texto, establecemos una relación directa entre patrimonio cultural y patrimonio artístico-histórico aún a riesgo de que resulte simplista y discutible. Es decir, pese a que la significación de la noción de patrimonio(s) ha ido variando en cada contexto histórico (Ballart, 1997; Prats, 1998; Hernández Hernández, 2002; González-Varas, 2003; Ballart y Juan, 2001; Ariño, 2002), sostenemos que el discurso hegemónico mantiene la misma concepción decimonónica de antaño en términos de apropiación y formación.

${ }^{6}$ El juego es sencillo: el continente contagia al contenido. Montaña es naturaleza, el pueblo está en la montaña, el pueblo es natural. Esta restitución tan simple ya ha sido adoptada por algunos municipios de montaña como una vía fácil de comunicación. Por ejemplo, en el parque natural de la Sierra Calderona (Valencia), el pueblo que mayor territorio aporta al parque ha adoptado como marca "Serra, tot natura". 
Con todo, y más allá de los discursos sobre lo in-material que configuran una realidad fragmentada, nos interesa destacar las praxis generadas en su edificación en un sentido amplio. La propia declaración del patrimonio intangible como Patrimonio de la Humanidad - en su primera versión Obras Maestras del Patrimonio Oral e Intangible de la Humanidad, en su segunda Lista Representativa del Patrimonio Cultural Inmaterial de la Humanidad - es un buen ejemplo para contemplar las asimetrías y jerarquías entre las distintas categorías patrimoniales que de forma muy significativa se han instituido como triada: material, inmaterial y natural. Asomarse a la lista de la UNESCO, cualitativa y cuantitativamente, deja patente dónde y porqué se sitúan los distintos patrimonios. La distinción bourdieana se plasma con fidelidad en la geografía física, política y simbólica de nuestro mundo. La propia UNESCO nos deja clara su visión normalizada del patrimonio mostrando el mapa, su mapa, de la distribución asimétrica de bienes en su ventana pública virtual (http:// whc.unesco.org). Y confiesa, a través de la Global Strategy for a Representative, Balanced and Credible World Heritage List aprobada por el Comité de Patrimonio Mundial en Thailandia (Phuket, 1994) ${ }^{7}$, la necesidad de establecer herramientas para elaborar una lista de patrimonio mundial más equilibrada y representativa ${ }^{8}$. Es más, declara sin tapujos, que la Lista del Patrimonio Cultural Inmaterial de la Humanidad ayudará a compensar el desequilibrio territorial porque permitirá a zonas sub-representadas - África, América y países del Pacífico-, tener un espacio en el mapa de la excelencia. Al respecto Kurin (2004a) señalaba que el programa de Obras Maestras del Patrimonio Oral e Intangible se vio como una posibilidad de compensar a las culturas del hemisferio sur. Y en el discurso del propio Koichiro Matsuura, en la primera proclamación de las Obras Maestras, es explicita esta idea?.

En este artículo, queremos presentar un análisis teórico/crítico de la construcción del patrimonio inmaterial en el contexto de la radicalización de la modernidad. En la última década, la producción de artículos sobre el patrimonio inmaterial, el número de reuniones internacionales que ha generado y el exponencial incremento en sus declaraciones nos hablan de un fenómeno vigoroso ${ }^{10}$. En cualquier caso, nuestro interés gira, sobre todo, en desenmascarar los campos de disputa contenidos en la institucionalización del patrimonio inmaterial como un espacio de producción de poder/saber (verdad/autenticidad) y, por tanto, de dominación. Para ellos presentamos, en primer lugar, una reflexión sobre las paradojas del término, con la intención de situar en un plano semántico las denotaciones y connotaciones que implica. Más allá de ser un juego discursivo, este ejercicio permitirá una primera aproximación a su nicho normalizado o naturalizado. El lenguaje no deja de ser un producto ideológico, en términos batjinianos un campo de lucha significativa. De ahí nuestro énfasis. En segundo lugar, daremos un paso más, atendiendo a su institucionalización

\footnotetext{
${ }^{7}$ Ver http://whc.unesco.org/en/sessions/18COM

${ }^{8} \mathrm{http}: / /$ whc.unesco.org/en/globalstrategy

${ }^{9}$ Ver fragmento del discurso en Aikawa (2004).

${ }^{10}$ Es importante tener en cuenta que esto no es exclusivo del patrimonio inmaterial, todos los procesos de patrimonialización están sometidos a inflación constante desde hace al menos
} cuatro décadas. 
y viendo cómo las distintas reglamentaciones han definido lo in-tangible como un conjunto de rasgos que es posible materializar. Así, nos acercaremos, de forma sintética, a lo sucedido en la declaración del Patrimonio Humanidad en lo referente al patrimonio inmaterial. Por último, abrimos algunas reflexiones sobre el peligro de volver a consolidar viejas prácticas colonialistas articuladas por un exacerbado neoliberalismo reduccionista.

\section{Desnudando la ortodoxia}

El propio término in-material, no-material, es paradójico y lleva consigo asociaciones cuanto menos sospechosas bajo la lógica de la racionalidad moderna -incorpóreo, irreal, abstracto, espiritual, ficticio, ideal, imaginario, invisible, sobrenatural, etcétera-. Del mismo modo, si preferimos optar por definirlo con el adjetivo de in-tangible nos encontramos con la misma trampa dialéctica: algo que no se puede tocar - impalpable, imperceptible, etéreo, sutil, sagrado, etcétera-. En realidad ambos remiten a la misma construcción de sentidos - performativos y normativos - y pueden intercambiarse sin mucho problema, es decir, en nuestra lengua podrían, aunque no lo son, funcionar como sinónimos - el primero remite a la no existencia material, el segundo a la imposibilidad de darle forma- De entrada sorprende que la contradicción inherente del término paradójicamente le dé sentido.

Con algo de sorna, podríamos decir que es entonces una cuestión de fe: no se ve, no se toca, pero es algo en lo que hay que creer - los antropólogos/as, sobre todo, por lo que nos atañe- . Pero más que fe, aunque sin duda unida a ella por el carácter espiritual y místico asignado ${ }^{11}$, habría que apuntillar, es una cuestión relativa al poder. Poder en su acepción más versada y sintética: como capacidad de generar verdad - autenticidad - a partir de la definición de la realidad. En este sentido, la categoría envía, de una parte, a los procesos constitutivos - genealógicos- de la ciencia moderna y, de otra, a la racionalidad por la que establecen procesos de purificación para la demarcación de lo posible - verdadero - a través tecnologías disciplinarias — no en vano subrayábamos nos atañe- Pese a que, desde el siglo XIX, las activaciones patrimoniales son paralelas — material, inmaterial, natural-, las demarcaciones han sido delimitadas gracias a prácticas e instituciones diferenciadas - espacios confinadores y normativas reguladoras - y alimentadas por la propia generación de conocimientos especializados — geo-epistemologías-.

Pero, ahora nos interesa seguir con las denotaciones del término para pasar después a las contradicciones. El concepto lleva al menos, de forma explícita, dos consideraciones que nos gustaría subrayar. Por un lado, se trata de concepto que, en su propia denominación, se define a través de la negación. Y la negación no deja de ser un vehículo que conlleva asociaciones inmediatas sobre aquello que de otro modo no podría ser. La entidad depende de su condición relacional (in-), por lo que su estatus epistemológico es, a todas luces, menor por su dependencia. Además, la negación siempre conlleva valoraciones negativas: denegación, prohibición, censura, desaprobación, etcétera. Por otro lado, en cuanto a la acepción de inmaterial

${ }^{11}$ Nótese aquí que este carácter es asimismo asignado a los patrimonios naturales. 
estaríamos hablando de un concepto desafortunado por las referencias ineludibles a las que remite el término no-material. Es decir, sería lo contrario a material. Y material se define ${ }^{12}$, si atendemos al diccionario, como un recurso, como algo real y efectivo y como útil. Todas estas adjetivaciones remiten a tener un valor intrínseco, por tanto, al negarlo ese valor propio desaparecería, siendo necesario la matización. De ahí que bajo nuestra consideración esté en desventaja al mostrarse como una suerte de negativo - de película — donde puede ser apresada el alma o el espíritu, pero donde no hay cabida para su exposición.

Frente al patrimonio material, que curiosamente se ve pero no se toca - principio fundacional sobre el que se cimienta la práctica museística de objetos sobresaturados de valor-, el inmaterial viene definido por su indefinición. Esto debería ser suficiente para andar con cierta cautela ¿Cómo es posible que se delimite algo sin fijar límites? ¿No es en sí mismo absurdo? La respuesta es clara, la vaguedad permite la entrada a toda suerte de interpretaciones lo que genera, de forma indirecta, consenso. En efecto, al ser una especie de cajón de sastre se da cabida a toda una suerte de manifestaciones naturalmente naturalizadas. Los pretendidos lindes ficticios establecidos entre lo material e inmaterial se desvanecen de forma constante tanto en nuestra propia cultura (Bouchenaki, 2003; Quintero, 2003; Van Zanten, 2004; Lacarrieu, 2008) como en comunidades donde no practican dicha distinción (Aikawa, 2004; Kurin, 2004a). O dicho de una manera más clara, como ya lleva tiempo apuntándose, ¿Pueden ser las catedrales sólo patrimonio material en cuanto belleza artística, prodigios de soluciones arquitectónicas y contención de objetos materiales valiosos - museísticos- o responden también a una concepción religiosa sobre el orden cósmico de nuestra práctica cultural? La pregunta ofende por estúpida. A pesar de ello, la elección de este ejemplo no ha sido aleatorio ${ }^{13}$ puesto que condensa los criterios eurocéntricos ${ }^{14}$ de la lista Patrimonio Cultural — material — de la Humanidad:

${ }^{12}$ Todo esto dejando de lado otras consideraciones filosóficas, económicas o políticas que en nuestro sistema hegemónico nos derivarían a debates entre el materialismo y el idealismo. Como señala Dubé "pensar en la in-materia (o en la no materia) es un ejercicio al que se entrega, desde hace siglos, una serie de ilustres filósofos, quienes, a través de los tiempos y las disciplinas, precisamente en eso parece que encuentren una materia inagotable de reflexión sobre la condición humana. Y con razón, de hecho el tema es inmensamente amplio y ha permitido desde siempre una panoplia de interpretaciones" (2004:125).

${ }^{13} \mathrm{Al}$ hacerlo queremos invertir la argumentación de Bouchenaki (2003), pese a que estamos de acuerdo con él parece que lo subordinado siempre requiera de explicación, de ahí que le demos la vuelta. Él toma como muestra para ilustrar la independencia entre el patrimonio material e inmaterial tres ejemplos de las declaraciones de Obras Maestras del Patrimonio Oral e Intangible de la Humanidad del 2001. Muchos autores siguen este modelo y ofrecen ejemplos al respecto.

${ }^{14} \mathrm{Al}$ respecto Dussel en una interesante reflexión habla de la confusión entre la universalidad y la mundialización implícita en el eurocentrismo: "Si se entiende que la 'modernidad' de Europa será el despliegue de las posibilidades que se abren desde su 'centralidad' en la Historia Mundial, y la constitución de todas las otras culturas como su 'periferia', podrá comprenderse el que, aunque toda cultura es etnocéntrica, el etnocentrismo europeo moderno es el único que puede pretender identificarse con la 'universalidad-mundialidad'. El 'eurocentrismo' de la Modernidad es exactamente el haber confundido la universalidad abstracta con la mundialidad concreta hegemonizada por Europa como "centro"” (2000:248). 
cristiandad, monumentalidad, occidentalidad e historicidad. Desde este plano, la materialidad e inmaterialidad tan sólo jerarquiza — cartografía político-cultural一, con todo lo que se desprende de esta inocente afirmación. Veamos otro ejemplo.

Sin duda, podemos darle la vuelta a esta fácil argumentación y formularla en otros términos que remitan a la materialidad ${ }^{15}$, en esta ocasión entendiendo la materialidad bajo un prisma económico - recurso para proceso productivo- ¿Pueden ser las fallas clasificadas como patrimonio inmaterial cuando mueven la friolera de setecientos cincuenta millones de euros ${ }^{16}$, si es que de valor hablamos? Más claro, las fallas estimulan mercados tan importantes como el turístico o el empresarial, generando tanto mano de obra estructural y puntual como sustanciales beneficios para la ciudad. No en vano se ha presentado el expediente para su declaración como Patrimonio Inmaterial de la Humanidad ${ }^{17}$, al mismo tiempo que se ha iniciado una interesante o interesada discusión sobre la necesidad de trasladar la festividad de San José a un día fijo - tercer lunes de marzo - para favorecer la llegada de turistas $^{18}$. Pero vayamos al grano, si el viejo debate planteado entre arte o artesanía, bien tratado por García Canclini (1993), se reduce a cuotas de mercado intercaladas y superpuestas con valoraciones - autenticidad - ${ }^{19}$, estaríamos ante un patrimonio material más que inmaterial por volumen de negocio y gestión cultural. Eso, claro está, dejando de lado los aspectos propiamente materiales de la fiesta por obvios -indumentaria, monumentos, instrumentos, pólvora, etcétera-. El problema, por tanto, estriba en el empeño de distinguir y catalogar a partir de criterios que no son operativos, lo que acarrea serios problemas teórico-metodológicos (Bortolotto, 2011a).

Ahora bien, detrás de ese empeño, es legítimo preguntarse qué se esconde, sobre todo, cuando se reconoce la clasificación como inoperante. En la Declaración de Estambul del $2002^{20}$, se decía expresamente, que existía un "lazo dinámico entre

${ }^{15}$ Aquí volvemos a dar una vuelta al planteamiento de Bouchenaki (2003), ya que en los tres ejemplos que pone hace referencia a criterios de monumentalidad (plaza o monumento histórico) o naturales (paisaje cultural). En nuestro caso, hemos optado por enfatizar el valor económico con una clara intención de relacionarlo con la autenticidad y, por tanto, con los mercados.

${ }^{16}$ Estudio realizado en el 2009 por la agrupación Interagrupación de fallas, presentado por la alcaldesa de Valencia.

${ }^{17}$ En 2011 se iniciaron los trámites para la declaración de las fallas como Patrimonio de la Humanidad. El día de la cridà de ese año (acto por el cual se da comienzo oficialmente a las fiestas falleras), la fallera mayor de Valencia se refirió ya a las mismas como Patrimonio de la Humanidad. En el 2012 la candidatura ha sido presentada y será evaluada por su admisión por la UNESCO en 2013.

${ }^{18}$ Hasta ahora la cremà, el día grande de la fiesta fallera, era el 19 de marzo. La llegada de visitantes siempre se ha relacionado con el día de la semana en que caía la festividad, este año al ser lunes se ha hablado de éxito turístico, titulares como "Un millón de turistas toma la ciudad" (El Levente, 18 de marzo del 2012) o "Lleno completo en Valencia (El País, 18 de marzo del 2012) lo reflejan. La propuesta para fijar el día no es nueva y lleva tiempo reivindicándose por la Federación Empresarial de Hosteleros de Valencia (El Levante, 21 de marzo del 2012).

${ }^{19}$ Autenticidad en relación con las valoraciones impuestas sobre atributos materiales (Munjeri, 2004).

${ }^{20} \mathrm{http}$ //www.lacult.org/docc/oralidad_11_10-11-declaracion-de-estambul.pdf. 
el patrimonio material e inmaterial y de profunda interdependencia" (punto 2); en el 2003, la $14^{\circ}$ Asamblea General de ICOMOS (Zimbabwe) se consagró al patrimonio inmaterial y su conferencia inaugural llevó por título "The interdependency of the tangible and intangible cultural heritage" (Bouchenaki, 2003); y en el 2004 la revista Museum International de la UNESCO dedicaba un número completo al patrimonio inmaterial. En él, numerosos expertos y asesores de distintas disciplinas valoraban el alcance de los progresos hechos en la UNESCO con el reconocimiento de nuevos patrimonios poniendo en evidencia los problemas conceptuales y prácticos de la denominación de inmaterial. Quizás atendiendo a su genealogía es posible atisbar esa resistencia a diluir los distintos patrimonios. Desde nuestra perspectiva, des-esencializarlos sería un ejercicio de subversión contra-hegemónico.

\section{Normativizando lo intangible como natural}

$\mathrm{Si}$ atendemos al despliegue y la consolidación de la institucionalización patrimonial en la esfera internacional, existen dos fechas claves, 1972 y 2003, que encapsulan, bajo nuestro punto de vista, las transformaciones más significativas experimentadas en la concepción del patrimonio en la posmodernidad. La primera, Convención sobre la Protección del Patrimonio Mundial, Cultural y Natural, simboliza la ruptura de la noción clásica del patrimonio — globalización patrimonialy el comienzo de la expansión de la cosmocultura ${ }^{21}$. Es decir, re-conoce y distingue entre bienes culturales y naturales como patrimonios comunes de la humanidad, de ahí la necesidad de custodiarlos. En este sentido, recordemos que no hay patrimonios sino 'patrimonializadores' (Montenegro, 2010) 22 . La segunda, Convención para la Salvaguarda del Patrimonio Cultural Inmaterial, equipara en 'calidad y carácter' a la anterior. $\mathrm{O}$ formulado de otro manera, establece treinta años después la igualdad conceptual — designación como Lista Patrimonio de la humanidad - y normativa - dotación de cuerpo jurídico - entre el patrimonio cultural, natural e inmaterial. En este proceso, es evidente que los recursos patrimoniales han dejado de ser, edificados o percibidos, como exclusivamente tangibles y materiales - culturales o naturales - ${ }^{23}$, pero aún así se han seguido aplicando las etiquetas.

De todas formas, el reconocimiento de otros bienes patrimoniales no es ninguna novedad. Lo que es novedoso es la forma de conceptualizarse y su pretendido reconocimiento universal. El llamado Folk-Lore ${ }^{24}$ y los primeros museos tradicionales al aire libre escandinavos (Santamarina, 2008) son muestras tanto de la patrimonialización temprana del llamado patrimonio inmaterial ${ }^{25}$, como de la edificación,

${ }^{21}$ Expresión tomada de Arizpe (2004).

${ }^{22}$ Esta afirmación, aparentemente sencilla, ayuda a aproximarse a la construcción de las geopolíticas.

${ }^{23}$ En este sentido, ha ocurrido lo mismo con la distinción entre bienes naturales y culturales.

${ }^{24}$ La paternidad del término anglosajón se debe a Thoms quién el 22 agosto del 1846 utilizó por primera vez folklore en una carta enviada a la revista The Athenaeum. Con ella definió un campo para los usos, costumbres y tradiciones populares. Desde entonces el concepto folclore fue utilizado como saber popular.

${ }^{25}$ Como señala Dubé (2004) en estos museos de artes y tradiciones populares, que abrieron una nueva museografía, y en ellos fue la concepción material la que prevaleció. 
división y categorización, entre la distinción de los tres patrimonios, todos vástagos de la primera modernidad. La necesidad del Estado Nación de articular un discurso homogéneo y legitimador sobre el territorio y la identidad desplegó distintas activaciones patrimoniales bajo criterios disciplinarios y científicos. Las demarcaciones políticas establecidas funcionaron como motores bien engrasados de conocimiento y reconocimiento — autenticidad —, provocando no sólo la jerarquización a partir de la asignación de valor sino también el olvido de lo — tratado - insignificante como mecanismo de distinción. Es más, en muchos casos, el olvido puede ser leído como una clara negación, sólo basta pensar en el patrimonio industrial y obrero (Castillo, 2004; Smith, 2011) o en el borrado sistemático de las patrimonializaciones naturales (Stevens, 1997; Santamarina, 2009). Resta decir que en esta primera configuración decimonónica, se evidencian ya todos los mecanismos reguladores que dibujarán décadas más tarde, y en sentido amplio, los mapas geopolíticos de la gestión cultural y del conocimiento. Esta herencia epistemológica mantenida hasta nuestros días, muestra una correlación estrecha entre objetos-sujetos y patrimoniables-patrimonializadores. Y, por ende, entre material-inmaterial.

Ahora bien, aunque situemos el re-conocimiento de los distintos patrimonios en el XIX, el camino de los tres fue divergente y respondió a lógicas y tiempos dispares. En el caso del patrimonio inmaterial, siguiendo a Kurin (1994a), a mediados del XX ya existe la voluntad de crear un instrumento jurídico internacional para su protección en dos sentidos. Por un lado, se inició la vía técnica y jurídica — derechos de autor en relación al folclore y la cultura popular-, y por otro, la vía nacionalista - políticas culturales-. En cualquier caso, los antecedentes son múltiples, pero es, sobre todo, a partir de la década de los ochenta ${ }^{26}$ del pasado siglo cuando empieza a constatarse una mayor atención al mismo. La Convención de Belgrado (1980), la Conferencia Mundial sobre Política Cultural celebrada en México (1982) o la creación en 1982 en la UNESCO del Comité de Expertos Gubernamentales en la Salvaguardia del Folclore son ejemplo de ello. Aunque podemos datar su arranque definitivo en 1989 con la Recomendación sobre la Salvaguarda de la Cultura Tradicional y Popular adoptada en París, el primer instrumento normativo para la salvaguardia del patrimonio inmaterial ${ }^{27}$. De la misma es interesante señalar que explícitamente se decía que "la cultura tradicional y popular forma parte del patrimonio universal de la humanidad" 28 . Con esta afirmación se abría la puerta a que los bienes inmateriales pudieran englobarse sin restricciones - aparentes- en el concepto de patrimonio mundial. La definición de cultura tradicional y popular suponía la superación, teórica al menos, del materialismo y monumentalismo imperante en la construcción del patrimonio. Además, la recomendación alentaba a los gobiernos a

${ }^{26}$ En la década de los 70 del XX había fracasado el primer intento de impulsar una protección para la cultura popular. La propuesta de Bolivia de 1973 para la protección y propiedad intelectual de las artes populares quedó en saco roto.

${ }^{27}$ Para un recorrido sobre la institucionalización en la UNESCO del patrimonio inmaterial se puede acudir a Aikawa (2009).

${ }^{28} \mathrm{http}: / /$ portal.unesco.org/es/ev.phpURL_ID=13141\&URL_DO=DO_TOPIC\&URL_SECTION=201.html 
impulsar investigaciones sobre cultura popular (Aikawa, 2009). A pesar del avance, el hecho de que fuera recomendación y se quedará tan sólo en un documento orientativo y no vinculante le restó importancia y muy pocos países atendieron a ella.

Una década después, en los noventa, según la institución internacional, la propia conciencia de las transformaciones glocales experimentadas y de los riesgos por la aceleración de los desplazamientos culturales hacía llevar al primer plano de su agenda lo inmaterial. En 1992 se creaba la Sección de Patrimonio Cultural Inmaterial de la UNESCO cuya primera presidencia ostentó la japonesa Noriko Aikawa (1992-2001). En 1993, gracias al acuerdo entre Japón y UNESCO se creaba el Fondo Fiduciario para la Salvaguardia del Patrimonio Cultural Inmaterial (Fundsin-Trust for the Preservation and Promotion of the Intangible Cultural Heritage). Este fondo fue establecido para subvencionar distintas actividades destinadas al fomento, educación y salvaguardia del patrimonio intangible y fue, tal y como reconoce la UNESCO, el mecenas de la Convención del $2003^{29}$. Japón desembolsó, hasta el 2007, 12 millones de dólares para este programa. En este sentido, tampoco puede ser casualidad que al final de la década, el presidente del Comité Mundial de Patrimonio de la UNESCO fuera el japonés Koichiro Matsuura, quien se convertiría, durante la siguiente década (1999-2009), en el Director General de la UNESCO. Sin duda, como señala Bortolotto (2010), Japón ha jugado un papel clave en la definición del paradigma de patrimonio inmaterial de la UNESCO y, podríamos añadir, que esto le ha situado en un mapa altamente competitivo, en los últimos años, quizás devolviéndole un protagonismo 'merecido' por la llamada razón de mercado (Ver gráficos 1 y 2). Por último, como señala Aikawa (2004), distintos factores contribuyeron en los noventa al reconocimiento del patrimonio inmaterial como: la Declaración de la Diversidad Biológica (1992), el Decenio de la ONU para las poblaciones Autóctonas y Minoritarias y el borrador de la Declaración de la ONU sobre derechos de los pueblos autóctonos — es importante notar y subrayar aquí la asociación que se desprende de lo inmaterial-.

En orden cronológico, en 1993, siguiendo la petición de la Republica de Corea, la UNESCO creaba el programa de Tesoros Humanos Vivos, invitando a los Estados miembros a impulsar este sistema. Un año después, la Declaración de Nara sobre la autenticidad (Japón, 1994) ${ }^{30}$, reclamaba una ampliación del concepto de autenticidad, estrechamente vinculado a lo material, y subrayaba la interrelación entre el patrimonio material e inmaterial. En cualquier caso, la conferencia advertía sobre los criterios occidentalistas de la UNESCO, denunciando el eurocentrismo contenido en la Carta de Venecia (1964), donde primaban los criterios de especialistas de la restauración-conservación. El concepto de autenticidad se vio como un modelo disciplinario exportado y frente a él el documento Nara reivindicó la autenticidad

${ }^{29}$ Ver http://www.unesco.org/culture/ich/index.php?pg=00115

${ }^{30}$ Ver http://www.esicomos.org/Nueva_carpeta/info_DOC_NARAesp.htm. Diez años después de esta conferencia, en Nara (2004) volvió a celebrar de nuevo una Conferencia Internacional sobre "La salvaguardia del Patrimonio Cultural Tangible y Intangible: hacia una planteamiento integrado". En ella se subraya que la autenticidad no es un término pertinente para el patrimonio inmaterial dado que el mismo está en constante recreación (Bortolotto, 2010). 
como una construcción social, reconociendo así el relativismo cultural del término (Bortolotto, 2010). La década se cerraba con la Consulta Internacional de Expertos sobre la preservación de Espacios Culturales Populares (1997) celebrada en Marraquech. En la misma se defendió la necesidad de crear una distinción de patrimonio oral e inmaterial, como Patrimonio de la Humanidad. Con esta aspiración, la consulta perseguía tener un reconocimiento para el patrimonio intangible homologable a la Lista del Patrimonio Mundial.

Desde nuestra perspectiva, esta conquista en el plano simbólico fue parcial. Si bien se conseguía que fuera un patrimonio distinguido como universal, la fórmula de la denominación y el procedimiento eran distintos por lo que la equivalencia no era total. El tan ansiado reconocimiento de la UNESCO, que buscaba enmendar la negligencia —omisión - , llegaba bajo la forma de Programa de Obras Maestras del Patrimonio Oral e Intangible de la Humanidad adoptado por el Consejo Ejecutivo de la UNESCO en 1998. Es interesante detenerse en las decisiones tomadas en cuanto al procedimiento para su declaración, dado que son representativas de las asimetrías cometidas, simplemente, atendiendo a su concepción reglamentaria. Destacamos tres: por un lado, las declaraciones se establecían cada dos años frente a las declaraciones anuales del patrimonio cultural/natural, lo que es indicativo de una desigualdad práctica y discursiva. No sólo porque, de forma implícita, sean menos importante sus declaraciones — no requieren atención anual— sino que por dicho procedimiento su visualización es menor - dispositivos de comunicación y marketing globalizadosPor otro, se limitaba el número de candidaturas por países, reduciéndola a una por cada declaración ${ }^{31}$, lo que confería un control directo sobre la cuantificación de las declaraciones ${ }^{32}$. Por último, pese a que esta distinción daba visibilidad a lo inmaterial no fue acompañada de un cuerpo normativo. De facto las indicaciones de Marraquech volvían a quedarse en recomendaciones, es decir, no había obligaciones.

En este sentido, y un año después de la distinción, la Conferencia Internacional Evaluación global de la Recomendación de 1989 sobre la salvaguardia de la cultura tradicional y popular: autonomía local y cooperación internacional celebrada en Washington (1999) llamaba la atención sobre la falta de un marco regulador. De ella interesa resaltar la petición explícita de no emplear la palabra folclore — por las asociaciones históricas negativas/peyorativas contenidas en su enunciado- proponiéndose en su lugar la fórmula de 'patrimonio cultural inmaterial'. Con esta recomendación se buscaba el consenso para salvaguardar la diversidad cultural y abría las puertas a la Declaración Universal de la UNESCO sobre la Diversidad Cultural (2001) donde se ponía el acento en la defensa de la diversidad cultural y la igualdad. La Mesa Redonda de Turín (2001) sobre las definiciones del patrimonio inmaterial y la posterior Mesa redonda sobre el patrimonio inmaterial y la diversidad cultural, que dio lugar a la Declaración de Estambul (2002), intentaron llegar a un acuerdo sobre los términos y mecanismos más apropiados para la futura convención del

\footnotetext{
${ }^{31}$ No así las trasnacionales, que eran ilimitadas.

${ }^{32}$ Ver http://unesdoc.unesco.org/images/0011/001142/114238s.pdf
} 
2003. El resultado: el consenso ${ }^{33}$ en detrimento de la complejidad y la ambigüedad (Khaznadar, 2009).

Desde nuestra consideración, todas estas recomendaciones, conferencias y declaraciones fueron las bases para el marco normativo de la Convención para la Salvaguardia del Patrimonio Cultural Inmaterial (París, 2003) que entró en vigor en el 2006. Antes de entrar en la misma es necesario subrayar el empleo del término salvaguardia en su enunciado en menoscabo del concepto protección aplicado generalmente en los textos jurídicos referidos al patrimonio. De algún modo esta sustitución se debe al reconocimiento de que el patrimonio material debe mantener sus condiciones frente a la naturaleza dinámica del inmaterial (Bortolotto, 2011a). En esta Convención se reconocía la asociación directa entre sitios, monumentos y museos con el patrimonio cultural y se subrayaba la necesidad de ver que "el patrimonio cultural comprende también el patrimonio inmaterial". El patrimonio cultural inmaterial, según la convención, lo constituyen los "usos, representaciones, expresiones, conocimientos y técnicas - junto con los instrumentos, objetos, artefactos y espacios culturales que les son inherentes- que las comunidades, los grupos y en algunos casos los individuos reconozcan como parte integrante de su patrimonio cultural. Este patrimonio cultural inmaterial, que se transmite de generación en generación, es recreado constantemente por las comunidades y grupos en función de su entorno, su interacción con la naturaleza y su historia, infundiéndoles un sentimiento de identidad y continuidad y contribuyendo así a promover el respeto de la diversidad cultural y la creatividad humana" (artículo 2.1.) No podemos detenernos en el análisis de la definición ${ }^{34}$, aunque de ella se desprenden cambios importantes con respecto a la recomendación de 1989, por ejemplo, la consideración del proceso frente a resultado, el acento en la identidad y la diversidad, el fomento al respeto o la relación entre los distintos patrimonios. Pero es interesante, al menos, atender a los tres rasgos que se asientan en la configuración final del patrimonio inmaterial de la humanidad en esta convención. El primero es la relación por oposición que se establece con el patrimonio material y natural, pese a la postura política mantenida de interdependencia, contenida incluso en la definición, se reafirma la disociación (listas separadas). El segundo es la indefinición — conjunto de expresiones-a través de características ambiguas y no equiparables - tradicional, oral e identitarioY el tercero es su carácter frágil — fugaz-, de ahí se supone la necesidad de protección - lógica del bien escaso y de valor-.

Mención aparte, por su calado, merece la polémica cláusula del 'respeto mutuo', que abría la caja de pandora. Cualquier bien para ser admitido en la lista debía cumplir unos requisitos: la sostenibilidad — paradójico, en cuanto no se sabe lo qué es y crea confusión sobre los criterios-, observar los derechos humanos - complejo, no se sabía hasta dónde interpretar - y el respeto mutuo entre comunidades — dificultoso, en la medida de establecer qué, por qué y para quién es ofensivo- . Por último, la equidad fue un valor suprimido, quizás porque se pensó como un imposible

${ }^{33} 120$ Estados miembro votaron a favor, algunos se abstuvieron y ninguno votó en contra.

${ }^{34} \mathrm{El}$ mismo daría para una artículo completo. 
— la discriminación de género, por ejemplo, planteaba dudas-. Estos requisitos nos devuelven al siempre complejo debate entre el relativismo extremo, el relativismo ético y el universalismo. No entraremos en él porque daría para mucho, preferimos cerrar observando algunos problemas conceptuales y prácticos de la convención.

A nuestro entender, pese al esfuerzo por subrayar la recreación constante "la dimensión evolutiva y procesual del patrimonio" (Bortolotto, 2011b) la convención establecía una imagen idealista y romántica de las praxis culturales, alejada de los procesos dinámicos de transformación, lucha y resistencia. Imagen, además, contradictoria porque se pretendía viva pero se instaba a congelarla (Kurin, 2004b, $2007)^{35}$, de ahí el salto hacia la salvaguardia. Una salvaguardia, siguiendo a Khaznadar (2009), que como instrumento normativo interviene en el devenir. La visión estática resultante, que recuerda a las viejas etnografías, niega o ataca, según se mire, a los propios cimientos de los sistemas culturales. Cabe preguntarse qué es lo que debe salvaguardarse, de forma forzosa, como vivo — ¿auténtico? - . Lo tradicional, bajo distintas versiones, vuelve a ser un comodín que permite amparar una disparidad de lógicas. Fórmulas como 'culturas vivas o vivientes' refuerzan el esquema de objetos muertos y nada más lejos bajo nuestra opinión. Las siguientes preguntas pueden sorprender pero detrás de ellas está la lógica oficial de las activaciones inmateriales: ¿hay tradición en peligro de extinción? ¿hay riesgo de perder lo auténtico? De ahí, que la propuesta resulte anacrónica al considerar "como inevitable la extinción de las culturas primitivas" (Bortolotto, 2011a: 29). Para nosotros, no hay ni praxis culturales ni objetos muertos, hay procesos, transformación, performances e intencionalidades. Y de todo ello se deriva la complejidad de encapsular en compartimentos lo inmensurable. Más si cabe cuando se delega, bajo la capa de la ética, la responsabilidad a las comunidades, conviertiéndose la participación en una muestra más de las paradojas y ambigüedades que existen tras la convención ¿Quién detenta la autoridad para la autentificación, clasificación y definición o los derechos y la propiedad cultural? ¿En quién recae la responsabilidad y en qué medida? O se pueden formular otras que ponen sobre la mesa las contradicciones ¿Quién reconoce la UNESCO como interlocutor? ¿En realidad no se produce una nacionalización de la cultura popular por un proceso de naturalización? (Kurin, 2007; Bortolotto, 2011b; Hafstein, 2011; Bendix, 2011; Noyes, 2011) ${ }^{36}$.

Todo ello sin detenernos ni en los problemas teóricos-metodológicos (Del Mármol, Roigé y Estrada, 2011) ni en lo que para Khaznadar (2009) es, sin duda, el defecto mayor de la convención: la jerarquía que establece entre las distintas expresiones culturales — ¿obras maestras?-. En este sentido se pregunta para quién son representativas las listas o de qué son representativas. La respuesta es obvia: para el discurso patrimonial autorizado, en términos de Smith (2011), que administra las

${ }^{35}$ Kurin se pregunta, en ese debate sobre la salvaguardia del patrimonio inmaterial tras la convención, si los museos pueden realmente conservar ese patrimonio. Desde su punto de vista este es el gran reto para los museos del siglo XXI.

${ }^{36}$ En este sentido, como apunta Hafsteis (2011), el reconocimiento de la UNESCO es una manera de reconocer las desigualdades, al reconocer que existen grupos que no poseen ni posibilidades económicas ni políticas. 
narrativas históricas y culturales para la legitimación, la negociación y mantenimiento de los valores y jerarquías culturales. El gobierno del pasado es clave para la definición y el control del presente.

\section{Del occidentalismo al orientalismo}

Antes de finalizar, nos gustaría acabar echando un vistazo a las declaraciones producidas para observar los deslizamientos producidos. Las primeras Obras de patrimonio inmaterial se declararon en marzo del 2001, fueron 19 espacios o formas de expresión relevantes, a los que se sumaron, en 2003, 28 obras. En el 2005, se produjo la tercera y última declaración bajo esta denominación incluyendo a 43 obras más ${ }^{37}$. Como vemos en cuatro años las declaraciones se doblaron, aunque en términos comparativos en 2005 el patrimonio inmaterial suponía tan sólo algo más del 10\% del conjunto del Patrimonio Cultural de la Humanidad. Hoy, tras el cambio de ser Obras a conformar Lista, los números no han variado en lo referido a la proporción ${ }^{38}$. En 2012 la Lista de Patrimonio Inmaterial de la Humanidad (PCI) la conforman 232 bienes las obras fueron integradas directamente y en el 2008 se incorporaron 90, en el 2009 otras 76, en el 2010 fueron 47 y en el 2011 las últimas 19-.

Vale la pena atender a la distribución geográfica y a los bienes declarados, porque nos hablan, más allá de las definiciones, de la praxis. Nos centraremos, de forma breve, en ello. En primer lugar, el mecanismo de la ubicación del PCI es parecido al del patrimonio natural y cultural: concentración y localización geográfica. Ahora bien, hay diferencias interesantes y notables en cuanto a confinación. Por ejemplo, tan sólo dos países, China (29 bienes) y Japón (20), concentran casi el 20 por ciento de los bienes inmateriales, o dicho de otra forma más clara, China tiene más bienes inmateriales declarados PCI que todo el continente africano (Ver gráfico 1) y casi

\section{COMPARATIVA DE LOS TRES PAÍSES CON MAYOR $\mathrm{N}^{\circ} \mathrm{DE}$ BIENES DECLARADOS}
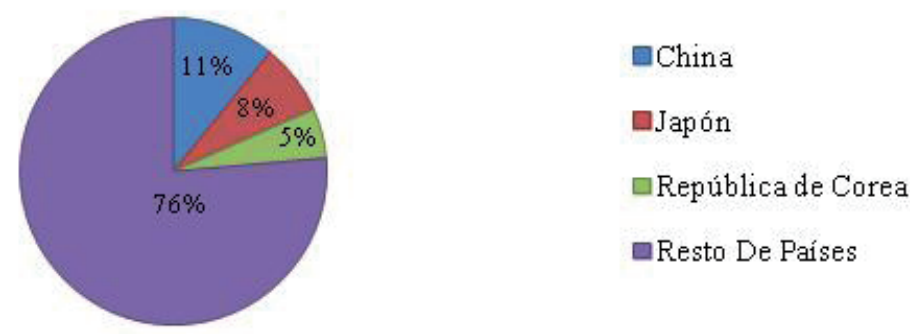

Gráfico 1. Comparativa de los tres países con mayor número de bienes inmateriales declarados PCI. Fuente: UNESCO. Elaboración propia. 2005.

${ }^{37}$ En nuestro país se reconocieron dos, el Misteri d'Elx en 2003 y la Patum de Berga en el

${ }^{38}$ Hasta la fecha, hay declarados 911 bienes en la Lista de Patrimonio Cultural y Natural de la Humanidad, 704 culturales, 180 naturales y 27 mixtos (Ver http://whc.unesco.org/en/list). 
los mismos que toda América. Esto sorprende en la medida en que el presidente de la UNESCO, como señalábamos al principio, veía en este patrimonio un instrumento para dar posibilidades de representación a los países en vías de desarrollo, pero se comprende por el desembolso japonés en el fondo para el PCI, el impulso de programas y conferencias en países orientales y la presencia nipona en órganos de decisión.

De hecho, si sumamos otros países orientales la representación de estos en la lista es de más del 50\% (India, 8, Mongolia, 8, Indonesia 5, etcétera) (Ver tabla 1). La tan acusada y denunciada occidentalización de la hasta ahora lista del patrimonio de la humanidad — casi el 50\% de los bienes están en Europa, estando Italia y España a la cabeza del top ten - que provocaba una dualidad entre países despatrimonializados frente a otros sobrepatrimonializados parece que quiera compensarse con la orientalización del patrimonio inmaterial (Ver gráfico 2). Los números hablan por sí solos, invirtiéndose los papeles y reproduciéndose el orden existente.

\section{BIENES DECLARADOS EN PAÍSES ASIÁTICOS}

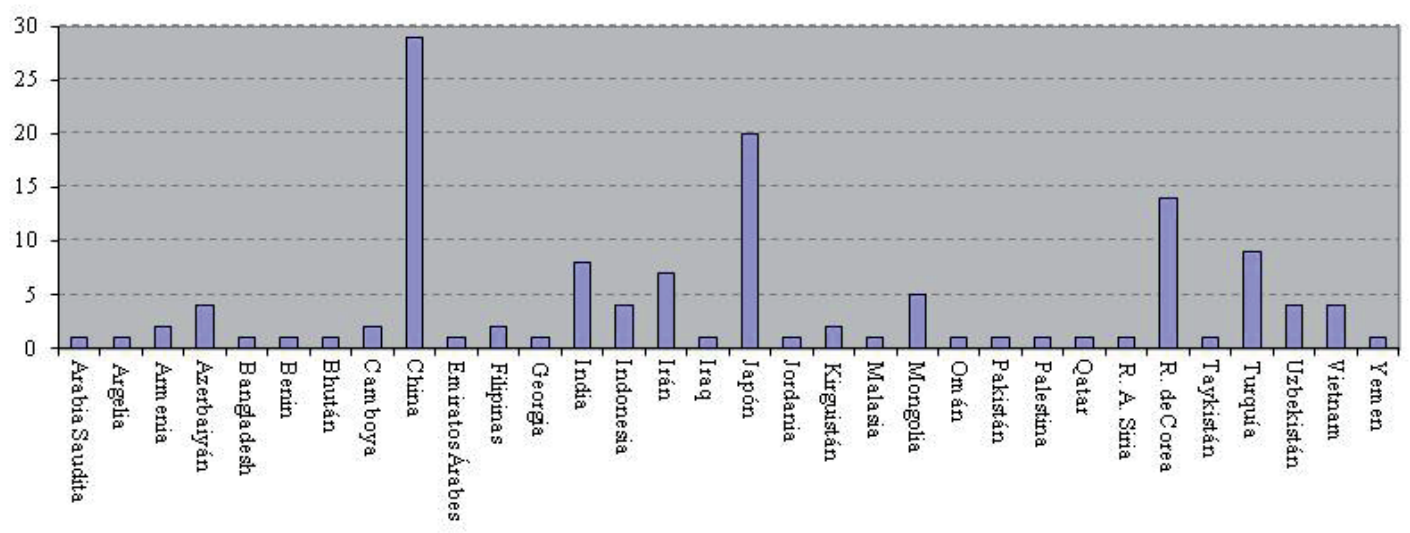

Tabla1. Bienes inmateriales declarados PCI en países asiáticos. Fuente: UNESCO. Elaboración propia.

Al final, nos encontramos con un mapa claro de las distribuciones - asimétricas - donde vemos cómo los distintos patrimonios - material, inmaterial y natural- aparecen especializados por zonas. De esta forma, se refuerzan los viejos desequilibrios colonialistas de antaño y se revitalizan los anacrónicos sistemas de clasificación y confinación para la domesticación naturalizada. La violencia simbólica contenida de estos mapas se edulcora, de tal manera que todos aplauden las distinciones en un ejercicio de mercados y valores confusos. Es decir, los procesos de mercantilización neoliberales se presentan tan normalizados —naturalizados y auténticos - que se adoptan y adaptan a las nuevas objetivaciones metaculturales en una suerte de ejercicio acrobático. En el mismo se hace patente la voluntad, la 
actualización y la persistencia por mantener revitalizadas las lógicas hegemónicas de las categorías. Al mismo tiempo, el PIC remite a procesos de mercantilización y comercialización, de ahí que se señalen sus riesgos (Hafstein, 2011).

\section{BIENES DECLARADOS POR CONTINENTES}
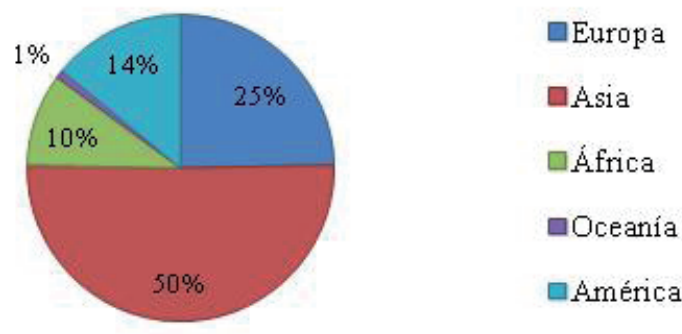

Gráfico 2. Bienes inmateriales declarados PCI por continentes. Fuente: UNESCO. Elaboración propia.

Y en esta dirección, y en segundo lugar, si atendemos a las categorías de inclusión para la declaración del PCI podemos intuir dos cosas. Por un lado, se manifiesta la complejidad y la dificultad de aplicar el sistema de catalogación del PCI. En la mayoría de bienes reconocidos, 75\% (Ver gráfico 3), su inclusión en la lista responde a más de una categoría de las cinco que se proponen (1.Tradiciones y expresiones orales, incluido el idioma; 2 . Artes del espectáculo: música, danza y teatro; 3 . Usos sociales, rituales y actos festivos; 4 . Conocimientos y usos relacionados con la naturaleza y el universo; 5. Técnicas artesanales tradicionales).

\section{$N^{\circ}$ DE CATEGORÍAS EN UN MISMO BIEN}

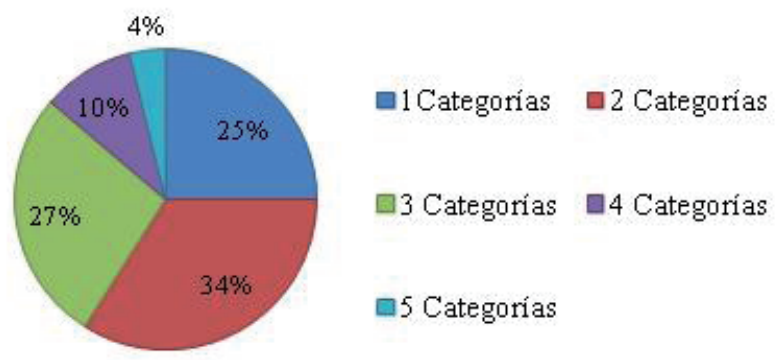

Gráfico 3. Número de categorías en un mismo bien en la lista PCI. Fuente: UNESCO. Elaboración propia. 
Y, por otro, si prestamos atención a los bienes declarados en una sola categoría podemos ver la lógica dominante de las declaraciones: el 70\% de las mismas recaen en la categoría 2 y 3 , espectáculos y rituales respectivamente (Ver gráfico 4). Siendo las 'tradiciones y expresiones orales, incluido el idioma' y los denominados 'conocimientos y usos de la naturaleza y el universo' casi insignificantes para el total de la lista, ambos suman el 5\%. Esto nos puede llevar a reflexionar en varios sentidos. De una parte, el criterio de materialidad o lo tangible sigue estando muy presente en esta lista. Es decir todo lo que se puede percibir - fotografiar- es mucho más fácil de incluir que aquello que no lo es. En realidad, lo que tradicionalmente se asociaba con el folklore sale reforzado, por lo que el giro en la concepción es limitado. De otra, se produce una jerarquía dentro de las propias categorías: primero, se sitúan las artes del espectáculo, después los rituales y, en tercer lugar, la artesanía. De tal forma que la lógica seguida es semejante a la practicada en el patrimonio material.

Veamos a lo que nos estamos refiriendo con una pequeña cata. Si atendemos por un momento a los bienes incorporados a la lista, en el 2008, de los países asiáticos - en aquel momento representaban el $41,5 \%$ del total de la lista- descubriremos que el $84 \%$ de los mismos responde a una categoría dominante, la $2^{39}$. Los bienes englobados suelen estar relacionados con el espectáculo - por dar algunos ejemplos: el teatro Wayang, teatro Kabuki, teatro Mak Yong, teatro Nôgaku, teatro kutiyattam, teatro Ningyo Johruri Bunraku, la ópera Kun Qu, la música del Morin Khuur, la música Shashmaqom, la música Nha Nhac, la música del duduk, la música del guqin, el canto védico, el canto de Sana'a, el canto polifónico georgiano, los cantos de los baul, los cantos Urtiin duu, los cantos épicos pansori, el Ballet Real de Camboya o la danza de Drametse, etcétera-. Sin duda, el teatro, la música y la danza son

\title{
CLASIFICACIÓN POR CATEGORIAS EN LOS BIENES CON UN ÚNICO REGISTRO
}

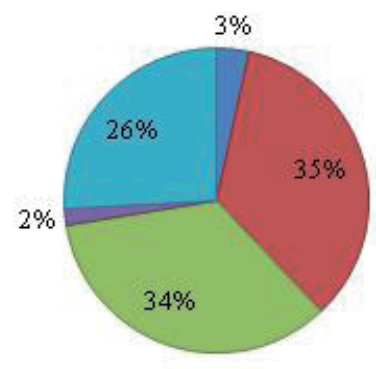

\author{
Expresiones Orales \\ Espectáculo: Música, Danza, Teatro \\ Usos Sociales, Rituales y Actos Festivos \\ - Conocimientos de la Naturalezay el Universo \\ Técnicas Artesanales Tradicionales
}

Gráfico 4. Número de categorías en un mismo bien en la lista PCI. Fuente: UNESCO. Elaboración propia.

${ }^{39}$ Salvo en una ocasión la categoría 2 se acompaña de otras. Generalmente de la 1 y la 3 . En este sentido, hemos contabilizado la categoría más repetida, asimismo hemos tenido en cuenta la denominación para ser inscrita. 
fáciles de ubicar en nuestro sistema de cognición ya que responden a espacios clásicos de 'distinción'. En definitiva, para aclararnos, es como si se jugara una partida donde los comodines ya están adjudicados. La apuesta por patrimonializar espacios culturales orientales parece remitir a iconos de poder occidentales - capitales puestos en circulación - que han sido constituidos como símbolos de exclusividad —la alta cultura- Resta explicar su eficacia práctica y discursiva.

\section{Conclusiones}

¿Es posible una perspectiva no eurocéntrica - globocéntrica - sobre el patrimonio? Probablemente no, porque sólo en el seno de una ideología reduccionista es posible producir procesos de depuración epistemológicos, de objetos y sujetos, para garantizar marcos de inteligibilidad afines; y con ellos, de producción y reproducción de un orden instaurado en la exclusión y la desigualdad práctica y discursiva.

La distinción del patrimonio inmaterial es un paso en el plano formal y en ese camino vemos los distintos giros practicados. En primer lugar, hemos pasado del discurso del proyecto nacionalista del XIX, al discurso global de las diferencias, es decir, el eje se ha desplazado de la distinción a la diferencia. En segundo lugar, hemos asistido a un cambio en la forma de protección pasando de la conservación a la salvaguardia. En tercer lugar, hemos presenciado una mudanza de los objetos a los sujetos o, si se prefiere, de los artefactos a los mentefactos ${ }^{40}$, con lo cual el acento pasa del resultado al proceso. En cuarto lugar, hemos pasado del confinamiento político-científico en la definición de lo patrimonializable al discurso que enfatiza la participación y la comunidad. Y, por último, todos estos cambios deben entenderse en el sutil deslizamiento hegemónico: del eurocentrismo hemos pasado al globocentrismo (Coronil, 2000). Un movimiento de ficha que oculta y legitima las asimetrías y los sometimientos. Visto así el andamiaje narrativo sobre el reequilibrio y la co-gestión del patrimonio inmaterial (Maguet, 2011) puede ser visto como un efecto perverso de la democratización (Noyes, 2011). En suma, por decirlo de una vez, si desvestimos lo inmaterial, descubriremos que bajo la nueva capa ceremonial se asoman los viejos ropajes de antaño. La ilusión óptica nos devuelve a la realidad fragmentada, China sigue siendo exótica, Kenia seguirá siendo salvaje e Italia siempre será Cultura.

\section{Referencias bibliográficas}

\section{AHMAD, Yahaya}

2006 "The Scope and Definitions of Heritage: From tangible to intangible", International Journal of Heritage Studies, 12: 292-300.

AIKAWA, Noriko

2004 "Visión histórica de la Preparación de la Convención Internacional de la UNESCO para la Salvaguardia del Patrimonio Cultural Inmaterial”, Museum International, 221: $140-153$.

${ }^{40}$ Tomo la distinción de Dubé (2004), pero añado a ella las asociaciones semánticas que diferencian los artefactos de los objetos. 
2009 "La Convention de l'Unesco pour la sauvegarde du patrimoine culturel immatériel e sa mise en œuvre" en VVAA. Le patrimoine culturel immatériel à la lumière del'Extrême-Orient, Paris, Maison des cultures du monde Babel, 13-45.

APPADURAI, Arjun

1996 La modernidad desbordada. Dimensiones culturales de la globalización, FCE, Argentina.

ARIÑO, Antonio

2002 "La expansión del patrimonio cultural" en Revista de Occidente, 250: 129-150.

ARIZPE, Lourdes

2004 "El patrimonio inmaterial, la diversidad y la coherencia", Museum International, 221: $133-140$.

ARIZPE, Lourdes

2006 "Los debates internacionales en torno al patrimonio cultural inmaterial", Cuicuilco, 13: 13-27.

BALLART, Josep

1997 El patrimonio histórico y arqueológico: valor y uso, Barcelona, Ariel.

BALLART, Josep y JUAN, Jordi

2001 Gestión del patrimonio cultural, Barcelona, Ariel.

BENDIX, Regina

2011 "Héritage et patrimoine: de leurs proximités sémantiques et de leurs implications" en Bortolotto, Chiara (Ed.) Le patrimoine culturel immatériel : enjeux d'une nouvelle catégorie, Paris: Éditions de la Maison des sciences de l'homme, pp. 99-121.

BORTOLOTTO, Chiara

2010 Globalising intangible cultural heritage ? Between internacional arenas and local appropriations" en Sophia Labadi and Colin Long (Ed). Heritage and Globalisation, London, Taylor \& Francis, 6-17.

2011a "Le trouble du patrimoine culturel immatériel" en Chiara Bortolotto (ed.) Le patrimoine culturel immatériel : enjeux d'une nouvelle catégorie, Paris: Éditions de la Maison des sciences de l'homme, 21-46.

2011 b "A salvaguarda do patrimônio cultural imaterial na implementação da Convenção da UNESCO de 2003”, Revista Memória em Rede, vol. 2, nº4.

BOUCHENAKI, Mounir

2003 "The interdependency of the tangible and intangible cultural heritage", http:// www.international.icomos.org/victoriafalls2003/papers/2\%20-\%20Allocution $\% 20$ Bouchenaki.pdf

BOURDIEU, Pierre

[1978](1998) La distinción. Criterio y bases sociales del gusto, Madrid: Taurus.

[1980](1991) El sentido práctico, Madrid: Taurus.

CASTILLO, Juan José

2004 "La memoria del trabajo y el futuro del patrimonio", Sociología del Trabajo, (52): 3-35. 
CORONIL, Fernando

2000 "Naturaleza del poscolonialismo: del eurocentrismo al globocentrismo" en Edgardo Lander (Comp). La colonialidad del saber: eurocentrismo y ciencias sociales. Perspectivas latinoamericanas, Buenos Aires, CLACSO, 87-111.

DEL MÁRMOL, Camila; ROIGÉ, Xavier; ESTRADA, Ferran

2011 "Safeguarding Intangible Cultural Heritage? A critical perspective on the inventory of Intangible Cultural Heritage in Catalonia" en Lira, Sérgio; Amoêda, Rogério; Pinheiro, Cristina (Coords.). Sharing Cultures. Portugal, Green Lines Institute for Sustainable Development, 481-490.

DUBÉ, Philippe

2004 "La hermosura de lo vivo o el regreso de lo reprimido", Museum International, 221: 125-132.

ESTEVE, Rafael

1983 Turismo ¿democratización o imperialismo?, Málaga, UMA.

FRIGOLÉ, Joan

2007 "Los modelos de lo rústico, lo salvaje y lo silvestre y la identidad de una valle del entorno de Cadí". En I. Vaccaro y O. Beltran (eds.), Ecología política de los Pirineos. Estado, Historia y Paisaje, Barcelona, Garsineu Edicions, 157-171.

FRIGOLÉ, Joan

2010 "Patrimonialization and the mercantilization of the authentic. Two fundamental strategies in a tertiary economy" en Roigé, X., Frigolé, J. (Eds). Constructing cultural and natural heritage. Parks, Museums and Rural Heritage, Barcelona, ICRPC, 27-38.

GARCÍA CANCLINI, N.

1993 "Los usos sociales del patrimonio cultural" En E. Florescano (comp.): El patrimonio cultural de México, México: FCE, 41-62.

GONZÁLEZ-VARAS, Ignacio

1999 Conservación de bienes culturales: teoría, principios y normas, Madrid, Cátedra.

HAFSTEIN, Valdimar

2011 "Célébrer les différences, renforcer la conformité" en Bortolotto, Chiara (ed.) Le patrimoine culturel immatériel: enjeux d'une nouvelle catégorie, Paris: Éditions de la Maison des sciences de l'homme, 75-97.

HEINICH, Nathalie

2009 La Fabrique du patrimoine. «De la cathédrale à la petite cuillère». Paris, Éditions de la Maison des sciences de l'homme.

HERNÁNDEZ HERNÁNDEZ, Francisca

2002 El patrimonio cultural: la memoria recuperada, Gijón: Trea.

HERNÁNDEZ I MARTÍ, Gil Manuel

2004 "La desterritorialització del patrimoni cultural en la modernitat globalitzada", El Contemporani, 41-47. 
HERNÁNDEZ, Gil; SANTAMARINA, Beatriz; MONCUSÍ, Albert; et al.

2005 La memoria construida. Patrimonio cultural y modernidad. Valencia, Tirant Lo Blanch.

HERZFELD, Michael

2004 The Body Impolitic: Artisans and Artifice in the Global Hierarchy of Value, Chicago, University of Chicago Press.

HOBSBAWN, Eric; RANGER, Terence

2002 La invención de la tradición, Barcelona, Crítica.

INIESTA, Montserrat

1994 Els gabinets del món. Antropologia, museus i museologies, Lleida, Pagès Editors.

KHAZNADAR, Chérif

2009 "Les dangers qui guettent la Convention de 2003" en VVAA. Le patrimoine culturel immatériel à la lumière del'Extrême-Orient, Paris, Maison des cultures du monde Babel, 101-109.

KIRSHENBLATT-GIMBLETT, Barbara

2004 "El patrimonio inmaterial como producción metacultural", Museum International, 221: 52-67.

KURIN, Richard

2004a "La salvaguardia del patrimonio cultural inmaterial en la Convención de la UNESCO de 2003: una valoración crítica", Museum International, 221: 68-81.

2004b "Museums and Intangible Heritage: Culture Deal or Alive?", ICOM News, 4: 7-9.

KURIN, Richard

2007 "Safeguarding of the Intangible Cultural Heritage: Key factors in Implementing the 2003 Convention", International Journal of Intangible Heritage 2: 10-20

LACARRRIEU, Monica (2008) “¿Es necesario gestionar el patrimonio inmaterial? Notas y reflexiones para repensar las estrategias políticas y de gestión”, Boletín Gestión Cultural, 17: 2-26.

LOWENTHAL, David

1998 El pasado es un país extraño, Barcelona, Akal.

MAGUET, Fredéric

2011 "L'image des communautés dans l'espace public" en Bortolotto, Chiara (ed.) Le patrimoine culturel immatériel: enjeux d'une nouvelle catégorie, Paris: Éditions de la Maison des sciences de l'homme, 47-73.

MARTÍ, Javier

2010 "Turismos y museos en la ciudad de Valencia" en ARRIETA, I (Ed.)

2010 Museos y parques naturales: Comunidades locales, administraciones públicas y patrimonialización de la cultura y la naturaleza, Bilbao, UPV, 63-90.

MONTENEGRO, Mauricio

2010 "La patrimonialización como protección contra la mercantilización: paradojas de las sanciones culturales de lo igual y lo diferente", Revista Colombiana de Antropología, 46: 115-131. 
MUNJERI, Dawson

2004 "Patrimonio Material e Inmaterial: de la Diferencia a la Convergencia, Museum International, 221: 98-109.

NASH, Dennison

1992 "El turismo considerado comouna forma de Imperialismo" en Smith, V (Ed.) Anfitriones e Invitados, Endymión, Madrid, 69-91.

NOYES, Dorothy

2011 "La fête ou le fétiche, le geste ou la gestion. Du patrimoine culturel immatériel comme effet pervers de la démocratisation" en Bortolotto, Chiara (Ed.) Le patrimoine culturel immatériel: enjeux d'une nouvelle catégorie, Paris: Éditions de la Maison des sciences de l'homme, 125-148.

PALUMBO, Berardino

2010 "Sistemi tassonomici dell'immaginario globale. Prime ipotesi di ricerca a partire dal caso Unesco" Meridiana, 68: 37-72.

PRATS, Llorens

1997 Antropología y Patrimonio, Barcelona, Ariel.

1998 "El concepto de patrimonio cultural”, Política y Sociedad, 27: 63-76.

QUINTERO, Victoria

2003 “El patrimonio inmaterial: ¿intangible?” en Hernández, Elodia y Quintero, Victoria (coord.) Antropología y patrimonio: investigación, documentación e intervención, Sevilla, Junta de Andalucía, 144-158.

QUINTERO, Victoria

2005 "El patrimonio intangible como instrumento para la diversidad cultural ¿una alternativa posible?" en Carrera, Gema y Dietz, Gunther (coord.), Patrimonio Inmaterial y gestión de la diversidad, Sevilla, IAPH, 68-84.

SANTAMARINA, Beatriz

2005 "Una aproximación al patrimonio cultural” en, G. Hernández, B. Santamarina, A. Moncusí y M. Albert: La memoria construida. Patrimonio cultural y modernidad, Valencia, Tirant Lo Blanch, 21-51.

2008 "De la educación a la interpretación patrimonial. Patrimonio, interpretación y antropología". En X. Pereira, S. Prado y H. Takenaka: Patrimonios culturales: educación e interpretación, Donosti, Ankulegi, 39-56.

2009 "De parques y naturalezas. Enunciados, cimientos y dispositivos, Dialectología y tradiciones populares, LXIV: 297-324.

SMITH, Laurajane

2011 "El Espejo Patrimonial. ¿Ilusión narcisista o reflexiones múltiples?” Antípoda, 12: 39-63.

STEVENS, Stan

1997 "The legacy of Yellowstone" en Stevens, Stan. (Ed.) Conservation through cultural survival: indigenous people and protected areas, Washington, Island Press, 13-32. 
VAN ZANTEN, Wim

2004 "La elaboración de una nueva terminología para el patrimonio cultural inmaterial", Museum International, 221: 36-43.

WEST, Paige y CARRIER, James

2004 "Ecotourism and authenticity", Current Anthropology, 45: 483-489. 